\title{
Engineered non-Mendelian inheritance of entire parental genomes in C. elegans
}

\author{
Judith Besseling \& Henrik Bringmann
}

\begin{abstract}
The ability to rewrite the rules of genetic segregation would open new possibilities in diverse areas of biotechnology ranging from breeding to epigenetics. Here we engineer non-Mendelian inheritance of the entire maternal or paternal genome in Caenorhabditis elegans by changing the structure of the mitotic spindle during the first cell division of the zygote. Using germline-specific overexpression of a single protein, the conserved microtubule force regulator GPR-1, we increase forces that pull on spindle poles to convert the single bipolar mitotic spindle to two monopolar spindles. This generates two-cell embryos in which one cell contains only the maternal chromosomes and the other cell contains only the paternal chromosomes. As the embryo develops, each cell of the animal, including the germ cells, contains the genetic material of only one parent, resulting in hybrid $F_{1}$ animals. Progeny of these animals $\left(F_{2}\right)$ inherit either only $F_{0}$ maternal or only $F_{0}$ paternal chromosomes, and thus descend from only either of their grandparents' gametes.
\end{abstract}

In sexually reproducing organisms, each parent makes an equal genetic contribution to the offspring. After formation of the zygote, separate pronuclei contain the alleles contributed by sperm and oocyte. In the course of the first cell division, chromosomes from both parents merge during mitotic spindle formation. Each chromosome is present as two sister chromatids, which are separated during anaphase and segregated to two nuclei that contain the same genetic information. Each nucleus then is passed to one daughter cell ${ }^{1}$. Microtubule-based forces that are generated by dynein and that are located on the cell cortex position the mitotic spindle ${ }^{2-5}$.

In C. elegans, microtubule-based pulling forces are controlled by a ternary complex consisting of G alpha proteins, LIN-5, and G-protein regulator 1 and 2 (GPR-1/2), which are two nearly identical regulators. GPR-1/2 has been shown to be required for force generation and potentially also force sensing to achieve spindle positioning and cytokinesis $^{6-11}$. The concentrations of GPR-1/2 are crucial determinants for microtubule-based pulling forces, as GPR-1 knockdown leads to reduced forces and GPR-1 overexpression leads to increased pulling forces in a dose-dependent manner ${ }^{12,13}$.

Here we use GPR-1 overexpression during the first cell division to change the rules of genetics. In our engineered C. elegans strains, each of the zygote's two progeny cells inherits one of the parental genomes, instead of a combined genome with contributions of both parents.
We propose that non-Mendelian genetics can be used for a wide range of applications ranging from studying genome regulation to breeding technologies and synthetic biology.

Our strategy was to increase pulling forces in the zygotic spindle to disrupt its normal formation by increasing the expression of the force regulator GPR-1, using codon adaptation-based overexpression. We used a synthetic gpr-1 transgenic insertion with a codon adaptation index of 1.0, driven by the pie-1 promoter, which resulted in germline-specific overexpression of GPR-1 (ref. 13; Supplementary Fig. 1). We then imaged the first cell division in these embryos using time-lapse microscopy and followed the segregation of chromosomes (Fig. 1a). In wild-type embryos, the paternal pronucleus, which had the two centrosomes attached, was positioned in the posterior. There, it met with the maternal pronucleus to form the pronuclear centrosomal complex (PCC). This complex then moved to the center of the embryo, and rotated so that the two centrosomes were aligned to the long axis of the embryo. After nuclear envelope breakdown a bipolar spindle formed, and the chromosomes were aligned in the metaphase plate. In anaphase, the sister chromatids separated and segregated into the two daughter cells.

In GPR-1-overexpressing embryos, the PCC formed normally in the posterior of the embryo. However, it did not center properly and did not align onto the long axis of the embryo. Instead, the PCC showed a vigorous rocking movement and was pulled apart before nuclear envelope breakdown, so that the two centrosomes were pulled to opposite poles of the embryo, each with one of the two pronuclei attached. As a consequence, two half-PCCs, each containing one pronucleus and one centrosome, were positioned at opposite ends of the embryo. Two monopolar spindles were formed in $70 \%$ of zygotes, whereas the remaining embryos formed bipolar spindles during first mitosis $(N=70, P<0.001$, compared with ten control embryos that did not carry the GPR-1 overexpression transgene and that all formed a bipolar spindle; Fig. 1a, Supplementary Movies 1-3, and Supplementary Figs. 2-4).

Typically, the paternal pronucleus was pulled to the posterior, and the maternal pronucleus was pulled to the anterior $(89 \%, N=18, P<0.05$, compared with a hypothetical 50\% distribution, Supplementary Movies 4 and 5). Each of the two half-PCCs then formed a monopolar spindle on either side of the embryo. During anaphase, the cytokinesis furrow separated the two monopolar spindles to form two daughter cells. The formation of two monopolar spindles appeared to be a unique constellation of zygotic mitosis, which is characterized by the

Max Planck Institute for Biophysical Chemistry, Göttingen, Germany. Correspondence should be addressed to H.B. (Henrik.Bringmann@mpibpc.mpg.de). 
a
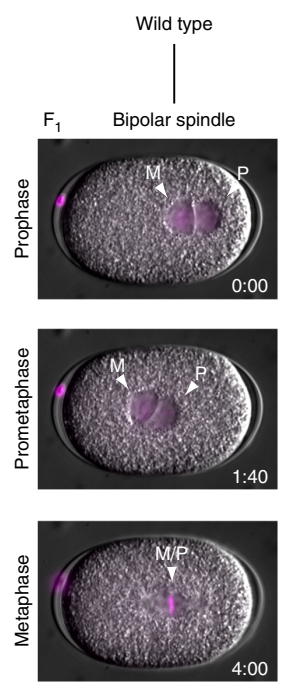

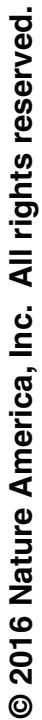
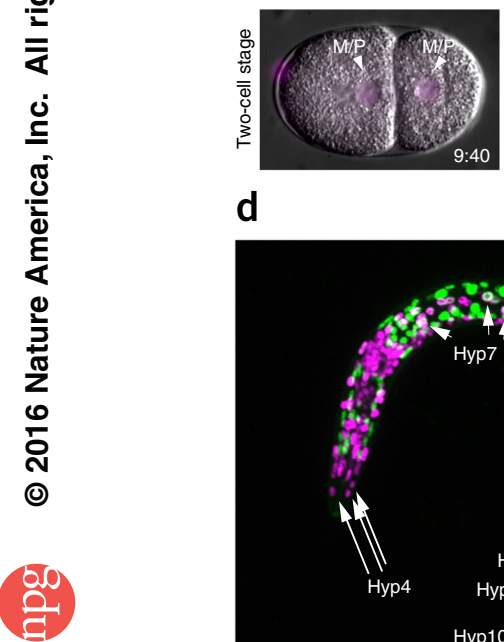

d
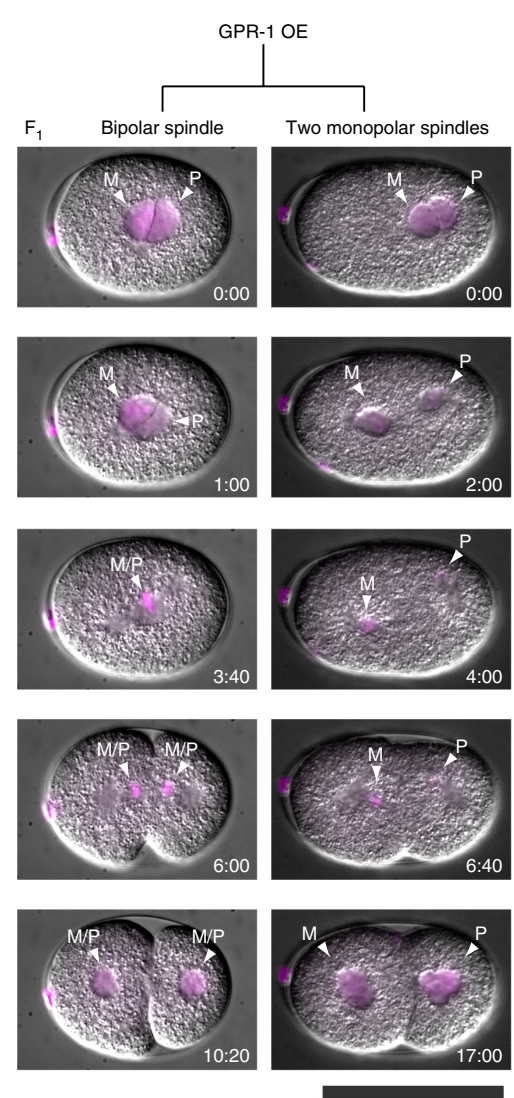

b

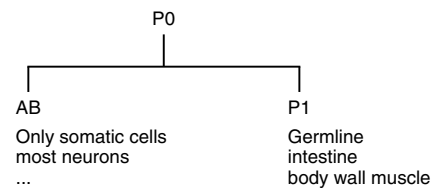

C
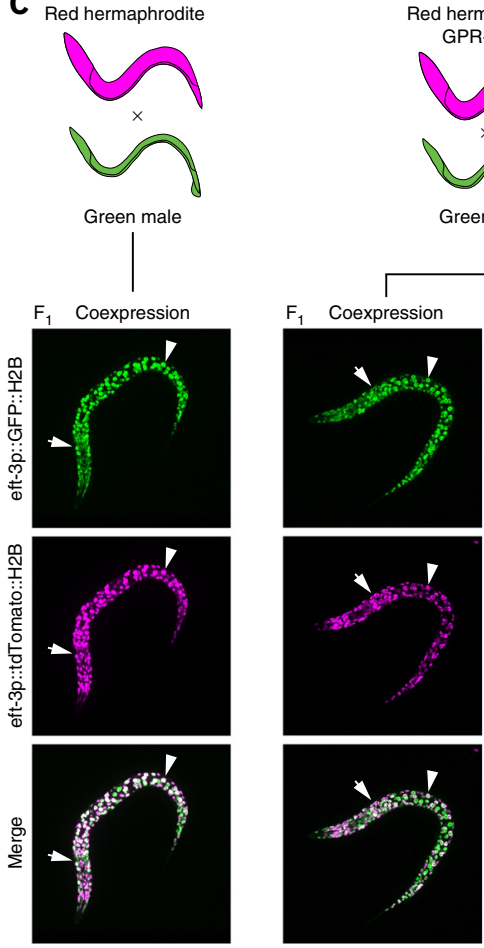

Separate expression
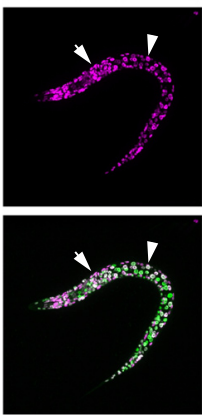
GPR-1 OE

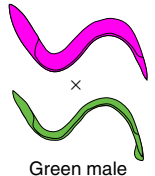

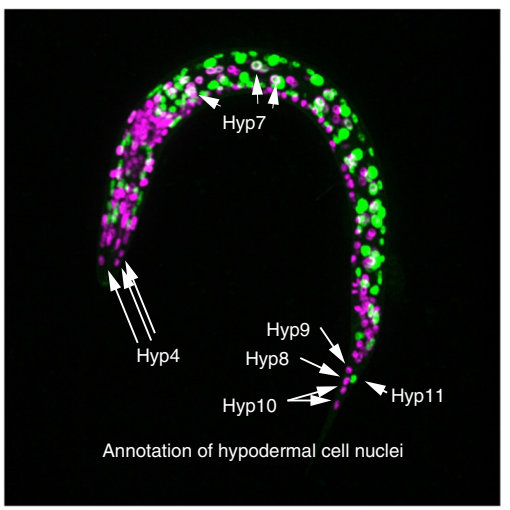
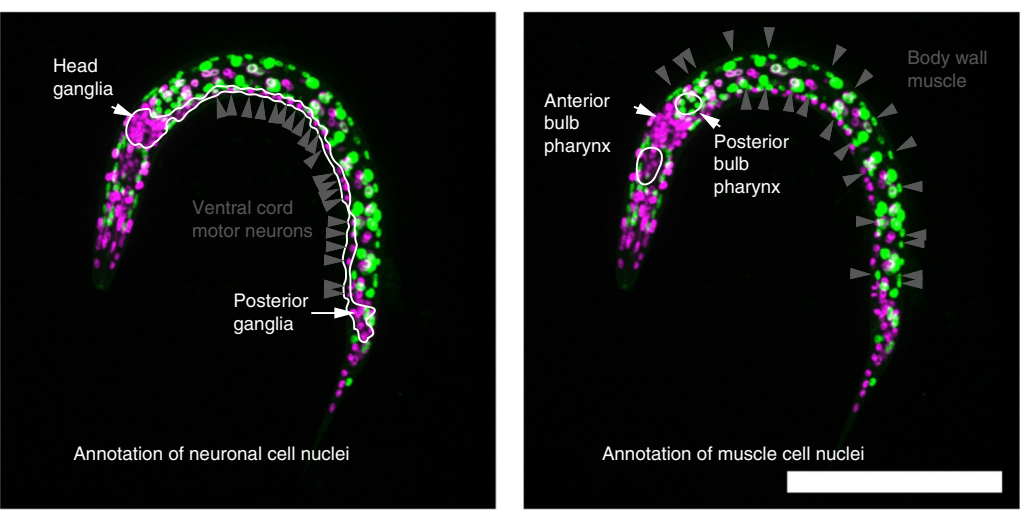

Figure 1 Increasing microtubule-based pulling forces leads to two monopolar spindles, which segregate chromosomes unequally. (a) Unequal segregation of chromosomes. Shown are combined DIC and fluorescence images. Left panel, the first cell division in wild-type embryos. DNA was stained by using mCherry:: histone expression (magenta). Paternal (P) and maternal (M) pronuclei met to form a pronuclear centrosomal complex. This complex moved to the center of the cell, where the mitotic spindle was formed and the DNA from both parents was merged in the metaphase plate, allowing an equal segregation of DNA in anaphase and thus an equal segregation of chromosomes to both daughter cells. Middle, formation of a bipolar spindle in GPR-1-overexpressing (OE) embryos. Right, formation of two monopolar spindles in GPR-1-overexpressing embryos. Note that the paternal and the maternal pronucleus met, but later separated. Each centrosome and pronucleus then formed a monopolar spindle, and paternal and maternal DNA was segregated to different daughter cells. The daughter cells often contained multiple small nuclei, perhaps resulting from the decreased confinement of the chromosomes in the monopolar spindle. Middle column: The remaining embryos formed bipolar spindles. Scale bar, $50 \mu \mathrm{m}$. Time is min:s. Two monopolar spindles were formed in 70\% of zygotes of GPR-1 over expressing embryos ( $N=70$ GPR-1 over expressing embryos, $P<0.001$, Fisher's exact test, compared with ten control embryos that did not carry the GPR-1 overexpression transgene and that all formed a bipolar spindle. For statistics, embryos without mCherry expression were used). (b) After the first cell division of the PO zygote, the two daughter cells AB and P1 develop into different lineages, AB into only somatic, and P1 into germline as well as somatic tissues, including intestine and body wall muscle. (c) After unequal chromosome segregation. Left, a control mating without GPR-1 overexpression. Right, crossing hermaphrodites that overexpress GPR-1 and ubiquitously express tdTomato-labeled histones (magenta) with males that ubiquitously express GFP-labeled histones (green) led to two types of progeny: individuals that co-expressed both markers in most cells and individuals that expressed the two markers separately in different tissues. Example animals shown using spinning disc microscopy. Arrows, head neurons; arrowheads, intestinal nucleus. Shown are animals at the larval stage L1. Scale bar, $100 \mu \mathrm{m}$. (d) In individuals expressing markers separately, the expression pattern matches the known cell lineage. Cell nuclei of the hypodermal cells, neurons, and muscles are annotated. Scale bar, $100 \mu \mathrm{m}$. Shown are the same microscope images as in c. 
Table 1 Inheritance of recessive mutations demonstrates non-Mendelian inheritance of entire genomes

\begin{tabular}{|c|c|c|c|c|c|c|c|}
\hline & \multirow{2}{*}{$\begin{array}{l}F_{1} \\
F_{2}\end{array}$} & \multicolumn{2}{|c|}{ Coexpression of markers } & \multicolumn{4}{|c|}{ Separate expression of markers } \\
\hline & & $\begin{array}{c}\text { Phenotypic mix containing } \\
\text { no mutants } N(\%)\end{array}$ & $\begin{array}{c}\text { Phenotypic } \\
\text { mix + mutants } N(\%)\end{array}$ & $\begin{array}{l}100 \% \text { GFP no } \\
\text { mutants } N(\%)\end{array}$ & $\begin{array}{l}100 \% \text { GFP + } \\
\text { mutant } N(\%)\end{array}$ & $\begin{array}{l}100 \% \text { Tomato no } \\
\text { mutant } N(\%)\end{array}$ & $\begin{array}{c}100 \% \text { Tomato }+ \\
\text { mutant } N(\%)\end{array}$ \\
\hline \multicolumn{8}{|l|}{ Paternal genotype } \\
\hline dpy-5(-) I/+; oxTi75 // & & $24(62)$ & $15(38)$ & $22(56)$ & $15(38)$ & $2(5)$ & $0(0)$ \\
\hline oxTi75 /I & & $4(100)$ & $0(0)$ & $24(96)$ & $0(0)$ & $1(4)$ & $0(0)$ \\
\hline oxTi75 II; dpy-17(-) III/+ & & $10(43)$ & $13(57)$ & $20(42)$ & $26(55)$ & $2(4)$ & $0(0)$ \\
\hline oxTi75 II; bli-6(-) IV/+ & & $8(62)$ & $5(38)$ & $15(36)$ & $26(62)$ & $1(2)$ & $0(0)$ \\
\hline oxTi75 II; dyp-11(-) V/+ & & $5(50)$ & $5(50)$ & $17(50)$ & $16(47)$ & $1(3)$ & $0(0)$ \\
\hline oxTi75 II; Ion-2(-) X/O & & $0(0)$ & $8(100)$ & $0(0)$ & $14(100)$ & $0(0)$ & $0(0)$ \\
\hline
\end{tabular}

The corresponding crossing scheme can be found in Figure 2. Some $F_{1}$ worms were selected for further analysis and were grouped in different classes determined by expression pattern of the fluorescence markers in the $F_{1}$ and by the presence or absence of the recessive phenotype and fluorescent marker expression in the $F_{2}$. This table shows the numbers of each category of $F_{1}$ observed for the crosses described in A. For most recessive crosses, about half of the $F_{1}$ segregated the recessive marker and the other half did not. For Ion-2, which is located on the $\mathrm{X}$ chromosome, all $\mathrm{F}_{2}$ broods contained lon-2 animals. Percentages were calculated per Mendelian or per non-Mendelian population. $3 \%$ of non-Mendelian worms contained the maternal P1 lineage (7/202), which is significantly different compared with a hypothetical $50 \%$ distribution $(P<0.001$, Fisher's exact test).

presence of two pronuclei, as bipolar spindles formed in subsequent divisions (Supplementary Fig. 5). Thus, an increasing microtubulebased-pulling force can generate two monopolar spindles during the first cell division. Each monopolar spindle contained the chromosomes from either the maternal or the paternal pronucleus, which resulted in the segregation of paternal and maternal chromosomes to different daughter cells.

Abnormal spindle formation in the zygote typically causes embryonic lethality ${ }^{14}$. To test whether embryos can survive after monopolar spindle formation we recovered these embryos after imaging and found that $38 \%$ of them completed embryonic development and hatched (Supplementary Fig. 6). It is known that the two daughter cells of the zygote develop into different lineages with different fates. The anterior cell (called AB) will give rise to only somatic cells, including almost all neurons. The posterior cell (called P1) will form the germ line as well as somatic cells, such as those of the gut and body wall muscle ${ }^{15,16}$ (Fig. 1b). To follow the segregation of paternal and maternal chromosomes into the different lineages, we mated GPR1-overexpressing hermaphrodites that also ubiquitously expressed transgenic histone:: tdTomato with males that ubiquitously expressed transgenic histone::GFP. We then looked at the expression of tdTomato and GFP in $F_{1}$ larvae. Equal segregation of chromosomes during the first cell division should result in expression of both markers in all cells of the worms. By contrast, unequal segregation of chromosomes should result in cells that express only GFP or only tdTomato, and the expression pattern should clearly match the known lineage. We found both $\mathrm{F}_{1}$ worms that expressed the two markers simultaneously in all tissues and $F_{1}$ hybrid individuals that expressed red and green markers in different tissues, consistent with the GPR-1overexpressing strain producing not only embryos with bipolar but also embryos with two monopolar spindles.

The majority of worms with unequally segregated markers expressed the maternal marker in cells of the $A B$ lineage such as neurons and the paternal marker in the cells of the P1 lineage such as muscle. Identification of individual cells of the hypodermis, which is made by cells from both the $\mathrm{AB}$ and the $\mathrm{P} 1$ lineages, indicated a lineage-specific rather than a tissue-specific expression (Fig. 1b-d and Supplementary Figs. 7-9). In 3\% of unequally segregated markers, we found the expression pattern to be inverted, so that cells of the $\mathrm{AB}$ lineage expressed the paternal marker (Supplementary Figs. 10 and 11, and Table 1). This shows that in most cases of unequal segregation, the paternal chromosomes were segregated to the $\mathrm{P} 1$ lineage, and the maternal chromosomes were segregated to the $A B$ lineage. This is consistent with the location of the paternal pronucleus more toward the posterior in the one-cell embryo, which led to almost exclusive paternal chromosome segregation to P1 when two monopolar spindles were formed.

The fraction of viable cross progeny with separate marker expression varied between 18\% and 77\% (Supplementary Fig. 11). Animals with separate expression were never found in control worms that resulted from mating in the absence of GPR-1 overexpression ( $N=331$ for GRP-1 overexpression; $N=1,781$ for control; $P<0.001$ ). Thus, whereas lineage-specific expression could also be explained by lineage-specific silencing of the reporter transgenes, the most likely interpretation of this experiment is that maternal and paternal DNA was segregated differently into the $\mathrm{AB}$ and $\mathrm{P} 1$ lineages, with a strong tendency for the paternal DNA to enter the P1 lineage.

Embryos laid by GPR-1-overexpressing hermaphrodites had a mortality of $28 \%$, and mating with males increased the mortality to 63\% (Supplementary Fig. 11). Hermaphrodites are self-fertilizing, and sperm almost always contains an X chromosome. By contrast, half of the male sperm contains one $\mathrm{X}$ chromosome, and the other half contains no $\mathrm{X}$ chromosome, which result in hermaphrodite or male offspring, respectively ${ }^{17}$. The $\mathrm{X}$ chromosome is essential for viability, and male cells are viable because they contain one copy of the $\mathrm{X}$ chromosome, which is maternally derived ${ }^{18}$. The unequal segregation pattern should result in a lineage that is devoid of any $\mathrm{X}$ chromosome, if the oocyte is fertilized with sperm that does not carry an $\mathrm{X}$ chromosome, and we would expect that such animals are not viable. Consistent with this idea we never found fertile males that expressed the markers in different tissues. Thus, the presence of an X chromosome appears to be essential for generating viable hybrid worms. Lethality reduces the number of offspring obtained, and using only $\mathrm{X}$-containing sperm increases the fraction of viable offspring.

The $\mathrm{P} 1$ lineage, but not the $\mathrm{AB}$ lineage, gives rise to the germline. Thus, an unequal segregation of chromosomes during the first cell division should result in non-Mendelian inheritance. We looked at the segregation of genotypes in the $\mathrm{F}_{2}$ generation after mating our GPR1-overexpressing strain that carried the red fluorescent marker with males carrying a green fluorescent marker. As expected, those $F_{1}$ that expressed both markers in all cells segregated offspring with a mix of genotypes containing either just one of the two markers, or both. However, those worms that expressed the markers in different tissues segregated only one marker. Of these, all individuals that expressed the paternal marker in the $\mathrm{P} 1$ lineage produced only $\mathrm{F}_{2}$ offspring with the paternal marker, that is, all $\mathrm{F}_{2}$ individuals expressed the paternal marker. All individuals that expressed the maternal marker in the P1 lineage produced only offspring with the maternal marker, that is, all $\mathrm{F}_{2}$ individuals expressed the maternal marker (Fig. 2a, Table 1, 
Figure 2 Unequal chromosome segregation leads to non-Mendelian inheritance of entire genomes. (a) Crossing scheme to follow non-Mendelian genetics. GPR-1-overexpressing (GPR-1 OE) hermaphrodites that expressed tdTomato histones ubiquitously were crossed with males that expressed GFP histones ubiquitously. $F_{1}$ individuals that co-expressed both markers segregated mixed genotypes as expected. Mosaic $F_{1}$ individuals that expressed the markers separately segregated only $F_{2}$ animals that were homozygous for only one parental marker. The majority of mosaic animals expressed the maternal marker in the nervous system and the paternal marker in body wall muscles, the intestine and the germline. These animals produced exclusively progeny homozygous for the paternal marker and lacking the maternal marker completely. $F_{1}$ mosaic animals with an inverted pattern were rare but produced only progeny that were homozygous for the maternal marker. (b) Crossing scheme for nonMendelian genetics using several genetic loci on different chromosomes. The fluorescent markers were always co-segregated with easily scorable genetic loci, which were on different chromosomes. Corresponding data can be found in Table 1 and Supplementary Figure 12. Thus, non-Mendelian segregation appeared to affect the entire genome.

and Supplementary Figs. 12 and 13). Thus, unequal segregation of genomes in the zygote results in non-Mendelian inheritance.

To test whether non-Mendelian inheritance affects only individual genes or entire genomes, we followed the segregation of two genetic loci that were located on two different chromosomes. A nonMendelian co-segregation of two unlinked loci would indicate that it is the entire genome that is segregated in a non-Mendelian fashion. We combined the inserted GFP transgene that we used above with alleles that cause recessive phenotypes. We selected classic mapping alleles that cause clear and easily identifiable phenotypes when homozygous such as dumpy $(d p y)$, blistered (bli), and long (lon). We selected one allele for each chromosome. Males were generated that were homozygous for the GFP insertion but were heterozygous for the recessive mutations (we kept the recessive alleles heterozygous because the homozygous phenotypes included low male fertility). We then crossed these males with hermaphrodites that were overexpressing GPR-1 and also carried a red fluorescent transgenic insertion as described above. We singled out $\mathrm{F}_{1} \mathrm{~s}$ and scored them as either co-expressing the two fluorescent marker genes or expressing them in different lineages. We then scored the genotypes of the offspring by checking for fluorescence and the recessive phenotypes. Each of the $\mathrm{F}_{1}$ worms that co-expressed the two fluorescent markers produced $\mathrm{F}_{2}$ worms with a mix of phenotypes including about one-quarter of worms carrying the recessive phenotype as expected for Mendelian inheritance. By contrast, each of the $F_{1}$ worms that expressed the paternal marker in the $\mathrm{P} 1$ lineage produced $\mathrm{F}_{2}$ broods that consisted of only one type of progeny. In about half of these paternal-only $\mathrm{F}_{2}$ broods, all individuals expressed the paternal GFP marker but did not show any recessive phenotype. In the other half, all animals also showed the respective recessive phenotypes. In the case of the $\mathrm{X}$-linked lon-2(-) locus, all individuals of the $\mathrm{F}_{2}$ broods showed the recessive phenotype. Thus, genetic loci on all six nuclear chromosomes showed co-segregation in a non-Mendelian fashion in the set of zygotes that resulted in a paternal P1 lineage (Fig. 2b, Table 1, and Supplementary Figs. 12 and 13). Thus, entire genomes appear to be segregated in this non-Mendelian inheritance.

Non-Mendelian inheritance could be used to study epigenetic regulation such as parent-of-origin effects ${ }^{19}$. Such effects likely exist in C. elegans but have been difficult to study owing to a lack of methods ${ }^{20,21}$. Apparently, animals with these all-maternal and all-paternal tissues can survive and are fertile. Thus, non-Mendelian hybrid $\mathrm{F}_{1}$ animals can be used as a model to study, for instance, a nervous system derived a
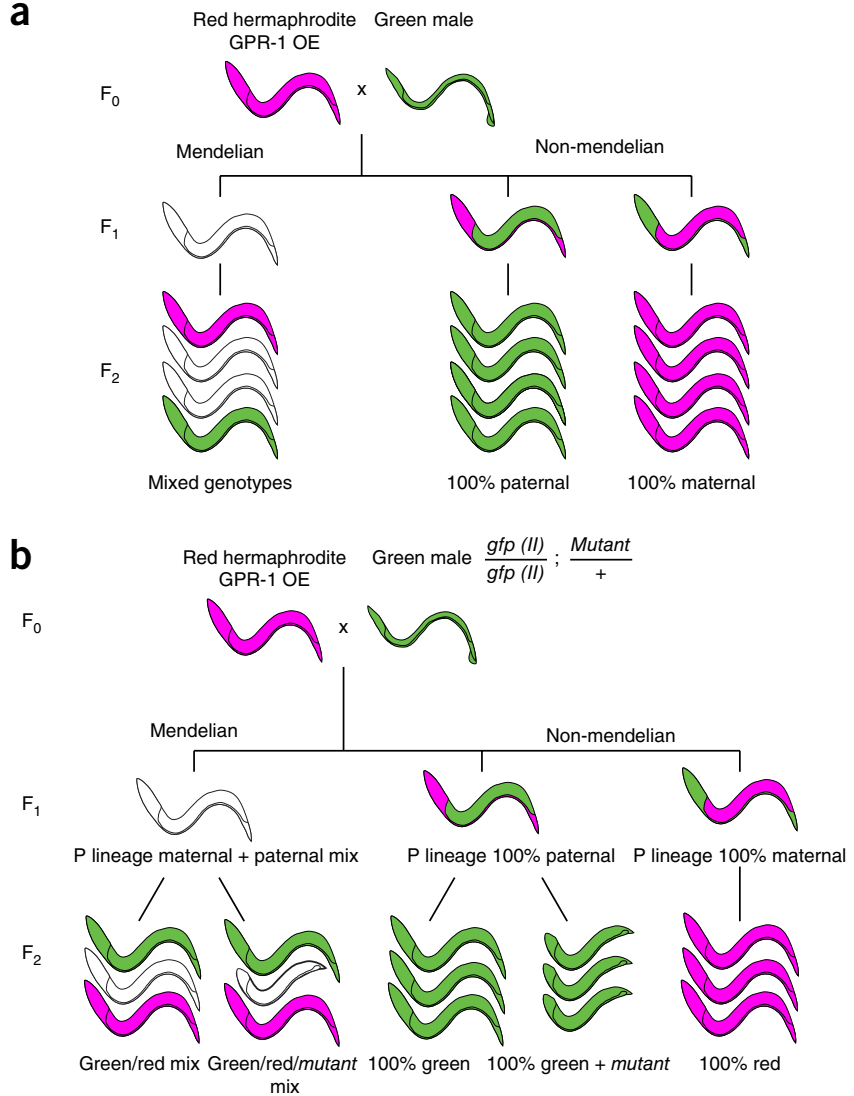

only from maternal chromosomes or an all-paternal gonad. Because the paternal and maternal genomes share a common cytoplasm in the zygote, non-Mendelian genetics could also be used to study epigenetic signaling between genomes and transgenerational inheritance ${ }^{22}$. For example, one could study whether and how epigenetic modifications of one genome affect the epigenetics of another genome. Molecular studies in this system would be straightforward, as the paternal epigenetic regulation can be compared before and after it was exposed to the environment of the maternal genome. Through these and other experiments, non-Mendelian inheritance will allow the study of the molecular biology of various epigenetic phenomena.

In addition to looking at genomic imprinting, non-Mendelian genetics can be used to generate intraspecies and maybe even interspecies hybrids. In intraspecies hybrids, genes can be manipulated specifically either in the $\mathrm{P}$ or in the $\mathrm{AB}$ lineage. For instance, lineagespecific knockouts can be generated. Interspecies hybrids may be generated between closely related species using non-Mendelian inheritance. Such hybrids certainly would differ from Mendelian interspecies hybrids and could be useful for studying speciation. Other potential applications include the study of a cell lineage that is devoid of an X chromosome, or the selective transfer of mitochondrial DNA to a different genomic background. Taken together, we provide a method that is imminently useful in C. elegans, as it can be used for a number of novel studies such as those described above, and we expect that the field will develop additional creative uses for nonMendelian inheritance.

It would be interesting to engineer genetic inheritance in other organisms, too. GPR-1 and its ternary complex are highly conserved. GPR-1 contains a so-called GoLoco motif, with which it binds to and activates heterotrimeric $\mathrm{G}$ proteins by a receptor-independent mechanism. In Drosophila, the GoLoco-containing GPR-1 homolog 
is called Pins, and the mammalian homolog is called LGN/AGS3. In all of these systems, the ternary complex controls the position of the mitotic spindle by regulating cortical dynein ${ }^{4,5,23}$. Because of the high conservation of the mechanisms that control spindle positioning it is likely that similar approaches can be developed in other systems.

This work shows that we can edit the rules of genetic inheritance by targeting zygotic mitosis, and it may be the starting point of the novel field of synthetic zoology, where cells and animals with novel properties will be designed.

\section{METHODS}

Methods and any associated references are available in the online version of the paper.

Note: Any Supplementary Information and Source Data files are available in the online version of the paper.

\section{ACKNOWLEDGMENTS}

The Max Planck Society funded this work.

\section{AUTHOR CONTRIBUTIONS}

J.B. performed experiments, analyzed data, and edited the manuscript. H.B. conceived the study, performed experiments, analyzed data, and wrote the manuscript.

\section{COMPETING FINANCIAL INTERESTS}

The authors declare no competing financial interests.

Reprints and permissions information is available online at http://www.nature.com/ reprints/index.html.

1. Oegema, K. \& Hyman, A.A. in WormBook (ed. The C. elegans Research Community) http://dx.doi.org/10.1895/wormbook.1.72.1 (2006).

2. Nguyen-Ngoc, T., Afshar, K. \& Gönczy, P. Coupling of cortical dynein and G alpha proteins mediates spindle positioning in Caenorhabditis elegans. Nat. Cell Biol. 9, 1294-1302 (2007).

3. Couwenbergs, C. et al. Heterotrimeric G protein signaling functions with dynein to promote spindle positioning in C. elegans. J. Cell Biol. 179, 15-22 (2007).
4. Kotak, S., Busso, C. \& Gönczy, P. Cortical dynein is critical for proper spindle positioning in human cells. J. Cell Biol. 199, 97-110 (2012).

5. Rose, L. \& Gönczy, P. in WormBook (ed. The C. elegans Research Community) http://dx.doi.org/10.1895/wormbook.1.30.2 (2014).

6. Srinivasan, D.G., Fisk, R.M., Xu, H. \& van den Heuvel, S. A complex of LIN-5 and GPR proteins regulates $\mathrm{G}$ protein signaling and spindle function in $\mathrm{C}$ elegans. Genes Dev. 17, 1225-1239 (2003).

7. Colombo, K. et al. Translation of polarity cues into asymmetric spindle positioning in Caenorhabditis elegans embryos. Science 300, 1957-1961 (2003).

8. Gotta, M. Dong, Y., Peterson, Y.K., Lanier, S.M. \& Ahringer, J. Asymmetrically distributed C. elegans homologs of AGS3/PINS control spindle position in the early embryo. Curr. Biol. 13, 1029-1037 (2003).

9. Bringmann, H., Cowan, C.R., Kong, J. \& Hyman, A.A. LET-99, GOA-1/GPA-16, and GPR-1/2 are required for aster-positioned cytokinesis. Curr. Biol. 17, 185-191 (2007).

10. Bringmann, H. G protein regulator 1 (GPR-1) localizes to cortical sites of artificial mechanical indentation in Caenorhabditis elegans zygotes. Cytoskeleton (Hoboken) 69, 819-825 (2012).

11. Krueger, L.E., Wu, J.C., Tsou, M.F. \& Rose, L.S. LET-99 inhibits lateral posterior pulling forces during asymmetric spindle elongation in C. elegans embryos. J. Cell Biol. 189, 481-495 (2010)

12. Pecreaux, J. et al. Spindle oscillations during asymmetric cell division require a threshold number of active cortical force generators. Curr. Biol. 16, 2111-2122 (2006).

13. Redemann, S. et al. Codon adaptation-based control of protein expression in C. elegans. Nat. Methods 8, 250-252 (2011).

14. Sönnichsen, B. et al. Full-genome RNAi profiling of early embryogenesis in Caenorhabditis elegans. Nature 434, 462-469 (2005).

15. Sulston, J.E. \& Horvitz, H.R. Post-embryonic cell lineages of the nematode, Caenorhabditis elegans. Dev. Biol. 56, 110-156 (1977).

16. Sulston, J.E., Schierenberg, E., White, J.G. \& Thomson, J.N. The embryonic cell lineage of the nematode Caenorhabditis elegans. Dev. Biol. 100, 64-119 (1983).

17. Meyer, B.J. in WormBook (ed. The C. elegans Research Community) http://dx.doi. org/10.1895/wormbook.1.8.1 (2005).

18. Brenner, S. The genetics of Caenorhabditis elegans. Genetics 77, 71-94 (1974).

19. Feil, R. \& Berger, F. Convergent evolution of genomic imprinting in plants and mammals. Trends Genet. 23, 192-199 (2007).

20. Haack, H. \& Hodgkin, J. Tests for parental imprinting in the nematode Caenorhabditis elegans. Mol. Gen. Genet. 228, 482-485 (1991).

21. Sha, K. \& Fire, A. Imprinting capacity of gamete lineages in Caenorhabditis elegans Genetics 170, 1633-1652 (2005).

22. Sarkies, P. \& Miska, E.A. Small RNAs break out: the molecular cell biology of mobile small RNAs. Nat. Rev. Mol. Cell Biol. 15, 525-535 (2014).

23. Kotak, S. \& Gönczy, P. Mechanisms of spindle positioning: cortical force generators in the limelight. Curr. Opin. Cell Biol. 25, 741-748 (2013). 


\section{ONLINE METHODS}

C. elegans husbandry and strains used. Strains were maintained on NGM medium and were fed OP50 bacteria as described by Brenner ${ }^{18}$. Strains were kept at $25^{\circ} \mathrm{C}$ during maintenance and all crosses. We reasoned that forces acting on microtubules and GPR-1 expression could be increased during higher temperatures. The following strains and genotypes were used in this study.

TH79: unc-119(ed3) III; ddIs21 [yfp::gpr-1; unc-119(+)].

TH242: unc-119(ed3) III; ddIs32[yfp ::gpr-1(synthetic, CAI 1.0, artificial introns); unc-119(+)].

EG1020: bli-6(sc16) IV; $d p y-11(e 224) \mathrm{V}$; lon-2(e678) X.

EG8040: oxTi302 [eft-3p::mCherry ::tbb-2 3'UTR + Cbr-unc-119(+)] I; oxTi75 [eft-3p::GFP::H2B::tbb-2 3'UTR + unc-18(+)] II; oxTi411 [eft3p::TdTomato::H2B::unc-54 3'UTR + Cbr-unc-119(+)] unc-119(ed3) III; him-8(e1489) IV ${ }^{24}$.

CB7272: ccIs4251 I; mIs12 II; dpy-17(e164) III; frIs7 IV; uIs69 V.

EG1000: $d p y$-5(e61) I; rol-6(e187) II; lon-1(e1820) III.

RW 10226: itIs37 [pie-1p::mCherry::H2B::pie-1 3'UTR + unc-119(+)]; stIs 10226[his-72 pomoter HIS-24::mCherry translational fusion with let-858 $3^{\prime} U T R+$ unc-119(+) $]^{25}$.

HBR1280: oxTi75 [eft-3p::GFP::H2B::tbb-2 3'UTR + unc-18(+)] II.

HBR1281: oxTi411 [eft-3p::TdTomato::H2B::unc-54 3'UTR + Cbrunc-119(+)] III; ddIs32[yfp::gpr-1(synthetic, CAI 1.0, artificial introns)].

HBR1282: oxTi411 [eft-3p::TdTomato::H2B::unc-54 3'UTR + Cbrunc-119(+)] III; ddIs32[yfp::gpr-1(synthetic, CAI 1.0, artificial introns)]; itIs37 [pie-1p::mCherry ::H2B::pie-1 3'UTR + unc-119(+)].

HBR 1283: oxTi75 [eft-3p::GFP::H2B::tbb-2 3'UTR + unc-18(+)] II; oxTi411 [eft-3p::TdTomato ::H2B::unc-54 3'UTR + Cbr-unc-119(+)] III.

HBR1284: $d p y-5(e 61)$ I; oxTi75 [eft-3p::GFP::H2B::tbb-2 $3^{\prime} U T R+$ unc-18(+)] II.

HBR1285: oxTi75 [eft-3p::GFP::H2B::tbb-2 3'UTR + unc-18(+)] II; dpy-17(e164) III.

HBR1286: oxTi75 [eft-3p::GFP::H2B::tbb-2 3'UTR + unc-18(+)] II; bli-6(sc16) IV.

HBR1287: oxTi75 [eft-3p::GFP::H2B::tbb-2 3'UTR + unc-18(+)] II; dpy-11(e224) $\mathrm{V}$.

HBR1288: oxTi75 [eft-3p::GFP::H2B::tbb-2 $\left.3^{\prime} U T R+u n c-18(+)\right]$ II; lon-2(e678) X.

Transgenes in C. elegans are commonly silenced in the post-embryonic germline. The mechanism behind this silencing is similar to regulation of $\mathrm{X}$ chromatin assembly ${ }^{26}$. The insertion ddIs32 [yfp ::gpr-1(synthetic, CAI 1.0, artificial introns)] overexpressed YFP ::GPR-1 at variable levels, and the transgene had a tendency to fall transcriptionally silent (Supplementary Fig. 1). As a workaround to maintain overexpression, all worms that contained $d d I s 32$ were regularly monitored for $y f p$ expression and an increased force phenotype. To maintain this transgene, we picked individual L4 larvae onto fresh Nematode Growth Medium plates and waited $24 \mathrm{~h}$ until the worms had laid eggs. Then, the mother animal was euthanized and embryos were transferred to a microscope. One single-cell embryo was filmed using timelapse differential interference contrast (DIC) and fluorescence microscopy across the first cell division. If the embryo showed two monopolar spindles and clear YFP expression, the plate onto which the mother animal had laid eggs was kept for further maintenance of this strain. For the non-Mendelian crossing experiments worms were only taken from plates founded by a mother that overexpressed GPR-1. Because of the silencing effect, mating plates with GPR-1 OE that contained no non-Mendelian offspring were removed from all analyses.

To test for non-Mendelian segregation of recessive mutant alleles, $d p y$-5(e61) I, dpy-17(e164) III, bli-6(sc16) IV, dpy-11(e224) V, and lon-2(e678) X were first crossed into the oxTi75 background and homozygous lines were isolated. Then, homozygous oxTi75 males were used to generate males that were heterozygous for the recessive allele but homozygous for oxTi75. These males were then crossed with HBR1281. About half of the $\mathrm{F}_{1}$ segregated the recessive allele, which could be seen by scoring the recessive phenotypes in the $\mathrm{F}_{2}$ generation.

Crosses were inspected for fluorescent marker expression using a Leica MC165 fluorescence stereomicroscope equipped with a dual-excitation dual-emission filter set for GFP and Texas Red (Chroma), that allowed the simultaneous observation of red and green fluorescence.

Microscopy. For time-lapse movies, a Nikon TiE inverted microscope was used. The embryo was imaged every $20 \mathrm{~s}$. First, a DIC image was taken and then a fluorescence image (wide field) using a standard GFP or Texas Red filter set (Chroma) and LED illumination (COOLLED). Images were acquired at $1,000 \times(100 \times$ oil objective) using an Andor iXon EMCCD camera. For analyzing lineage-specific expression, larval stage L1 animals were anesthetized with Levamisole and were imaged using an Andor Revolution Spinning disc system (488- and 565-nm laser wavelengths).

Statistics. Fisher's exact test was used unless stated otherwise.

24. Frøkjær-Jensen, C. et al. Random and targeted transgene insertion in Caenorhabditis elegans using a modified Mos1 transposon. Nat. Methods 11, 529-534 (2014).

25. Murray, J.I. et al. Multidimensional regulation of gene expression in the $C$. elegans embryo. Genome Res. 22, 1282-1294 (2012).

26. Schaner, C.E. \& Kelly, W.G. in WormBook (ed. The C. elegans Research Community) http://dx.doi.org/10.1895/wormbook.1.73.1 (2006). 